\title{
A COMPARATIVE STUDY BETWEEN INTRAOPERATIVE CLINICAL AND POSTOPERATIVE RADIOLOGICAL MEASUREMENT OF DISTANCE BETWEEN TIP OF GREATER TROCHANTER TO CENTER OF FEMORAL HEAD IN OPERATED CASES OF HIP HEMIARTHROPLASTY
}

\author{
Hiranmoy Deb ${ }^{1}$, Sunny Kumar Mallick ${ }^{2}$
}

${ }^{1}$ Assistant Professor, Department of Orthopaedics, R. G. Kar Medical College, Kolkata, West Bengal, India.

${ }^{2}$ Consultant Orthopaedic Surgeon, Department of Orthopaedics, Guwahati Neurological Research Center (GNRC), Kadambagachi,

West Bengal, India.

\begin{abstract}
BACKGROUND

Hip joint is a polyaxial ball and socket joint. At different stages of gait cycle, joint reaction forces at the hip joint depend on abductor leaver arm which is function of neck length (Offset), neck shaft angle and body weight. During Arthroplasty operation of hip, femoral offset measurement and its restoration to anatomical limit is important. In our study, we have measured the femoral neck length, between two fixed bony points, from the tip of greater trochanter (GT) to the center of the femoral head peroperatively and again post-operatively, from the true size x-ray of the replaced femoral head. We compared this measurement with the postoperative x-ray of normal side hip, to compare, how far normal offset is achieved after operating with various prosthesis (fixed bipolar prosthesis and modular prosthesis) available in the Indian scenario.
\end{abstract}

\section{MATERIALS AND METHODS}

This is a prospective, comparative study on presentation at in-patient department (IPD). Total 53 patient (22 male and 31 female) above 60 years of age, admitted at our institution 'Ramakrishna Mission Seva Prathistan (VIMS)' in the year 2015-16, with intracapsular fracture neck femur of either right or left side were studied. Per-operative measurement was taken during hemiarthroplasty operation using fixed bipolar prosthesis or modular prosthesis (cemented/un-cemented) available in the Indian market. Post-operative measurement taken in the true size x-ray of two fixed points on the operated and normal side. Limb length was measured clinically. The values obtained were compared to establish how far the normal anatomical offset is achieved after hemiarthroplasty operation by using prosthesis manufactured by different companies in India.

\section{RESULTS}

Intraoperative measurement of distance between tip of GT and center of femoral head corresponded with the measurement taken from tip of GT to the prosthetic femoral head. This signifies that we have successfully corrected the offset with available prosthesis with correct surgical technique. There is no significant difference in the postoperative true size X-ray of distance between tip of GT to the center of prosthetic femoral head and the tip of GT with center of femoral head of normal side.

\section{CONCLUSION}

Our study results shows that by opting correct surgical technique and appropriate neck resection, currently available prosthesis (FBP \& MBP) in our country can achieve desired neck offset length in comparison to the normal hip and had no significant limb length discrepancy.

\section{KEY WORDS}

GT, FBP. MHDG MBP.

HOW TO CITE THIS ARTICLE: Deb H, Mallick SK. A comparative study between intraoperative clinical and postoperative radiological measurement of distance between tip of greater trochanter to center of femoral head in operated cases of hip hemiarthroplasty. J. Evolution Med. Dent. Sci. 2018;7(52):5543-5548, DOI: 10.14260/jemds/2018/1227

\section{BACKGROUND}

The hip joint is a multiaxial spheroidal ('Ball and Socket') joint, which allows relatively unhindered motion in three degrees of freedom, i.e. flexion/extension, abduction/ adduction, medial (Internal) and lateral (External) rotation. Under bipedal loading conditions, the femoral heads support the weight of the body minus the weight of both legs

'Financial or Other Competing Interest': None.

Submission 16-11-2018, Peer Review 11-12-2018,

Acceptance 17-12-2018, Published 24-12-2018.

Corresponding Author:

Dr. Sunny Kumar Mallick,

3/161, Jatindas Nagar,

PO \& PS-Belgharia,

District-North 24 Parganas,

Kolkata-700056, West Bengal, India.

E-mail: sunny.rgkmc@gmail.com

DOI: $10.14260 /$ jemds $/ 2018 / 1227$
(Approximately one-third body weight) and the resultant vectors are vertical. The factors influencing both the magnitude and direction of the compressive forces acting on the hip are the position of the centre of gravity; the abductor moment arm, which is a function of neck length (Offset) and neck-shaft angle; the magnitude of body weight.(1) To understand hip bio mechanics, the understanding of femoral offset measurement, (2) will be helpful to Orthopaedic surgeons during their total or hemi hip replacement operations.(3)

When we wanted to find data on femoral offset measurement, we found that there is data regarding measurement of femoral offset radiologically(4) and per operative measurement in western population(5) but no study state about the restoration of femoral offset after THR or hemiarthroplasty in Indian population.(6) For clinical measurement of femoral offset we chose two fixed bony points- 
1. Tip of greater trochanter,

2. Centre of femoral head in neutral position of hip joint.

In this study we attempted to find the per operative distance between tip of greater trochanter and centre of femoral head clinically.

And in postoperative hip of arthroplasty (Both hemi and total) patients in true sized skiagram the distance between tip of greater trochanter and centre of the head of replaced prosthesis is measured. Thus, we tried to find how much restoration of the distance between two fixed points is possible, after replacement of that hip joint.

To make a suitable femoral prosthesis design for Indian population.

\section{Objectives of the Study \\ Primary Objectives}

1. To measure the distance between tip of greater trochanter and centre of femoral head (in the line of femoral anteversion the greatest distance was taken), intra operatively.

2. To measure the distance between the tip of greater trochanter and centre of the head of femoral prosthesis, radiologically.

3. To measure the distance between tip of greater trochanter and centre of femoral head of normal side, radiologically.

\section{Secondary Objectives}

1. To correlate the anatomical hip's offset and replaced hip's offset.

2. To know the range of limb length discrepancy with change of offset.

\section{MATERIALS AND METHODS}

\section{Study Site}

Patients admitted in orthopaedic department of Ramakrishna Mission Seva Pratishthan (VIMS), Kolkata, in orthopaedic department after getting Institutional ethics committee.

\section{Study Population}

Patients will mostly come from Kolkata as well as eastern part of India with pathology in hip undergoing THR/ hemiarthroplasty.

\section{Study Design}

A prospective, comparative study on presentation at in patient department (IPD).

\section{Sample Size}

It is estimated that at least 32 subjects will be required for $5 \%$, A p-value is less than 0.05 it will be considered as significant. Using the following formula-

$\mathrm{N}=\sigma^{2}\left(\mathrm{Z}_{\alpha / 2}+\mathrm{Z}^{\beta}\right)^{2} /\left(\mathrm{d}^{2}\right)$ Where $\mathrm{N}$ is sample size

- $\sigma$ (standard deviation of the within-pair difference for distance between tip of greater trochanter and centre of femoral head ) taken as $2.3 \mathrm{~mm}$ (IJ0;Swiwatch\&Dahiya etal(2003); vol.37, issue 4 page $247-251)^{7}$

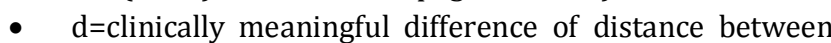
tip of greater trochanter and centre of femoral head taken as $1.13 \mathrm{~mm}$ (seen at par operative and postoperative)
- $\quad \mathrm{Z} \beta=$ corresponds to power $(.84=80 \%$ power $)$

- $\mathrm{Z} \alpha / 2=$ corresponds to two-tailed significance level(1.96 for $\alpha=.05$ )

But we take sample size as 53 patients as this number of patients were admitted in that one-year study period in our institution, who had matched with the inclusion criteria. In this period, we didn't have any patient, who underwent total hip replacement operation in our institution. So we collected data only from the patients, who underwent hemiarthroplasty.

Although in our methodology an optimum required sample size was 32 but we have taken 53 participant as we get this no of patient during this year(2015-16), so we had taken 53 as sample size for this study. So we had achieved more than optimum number for this study.

\section{Time Frame}

One year (June 2015 - May 2016)

\section{Inclusion Criteria}

Any patient having fracture neck of femur or pathology in hip, who needs total or hemi replacement of hip.

\section{Exclusion Criteria}

Any patient with deformed head or neck of femur and trochanteric region on either side.

\section{Method of Sampling}

A Simple random Sampling procedure was adopted to select the patient after his/her consent and within our inclusion criteria of the study.

\section{Methodology}

The study is carried out in the department of orthopaedic surgery at Ramakrishna Mission Seva Pratishthan (VIMS). After approval of institute ethical committee, we started the study. Complete medical and surgical check-up was performed in each patient including proper history taking, preoperative investigations etc.

- Written informed consent in patients' own language was obtained from all patients.

- After receiving the patient in operation theatre per operative measurement was taken.

- Post-operative radiograph was taken during first follow up visit.

\section{Operative Procedure}

After routine pre-operative investigation patient is posted for operation

After anaesthesia (Spinal/epidural/General), patient was positioned with sterile dressing and draping, posterior (POST) or modified Hardinge (MHDG) approach of the hip was used in order to access both the head and neck of femur and acetabulum. $[11,12,15,16]$

After head delivery without cutting the neck (As most of the cases we got are intracapsular fracture neck of femur), the following measurements were taken.

a) Diameter of Femoral Head: Femoral head gauge was used for this purpose.(8) It was measured after extracting out the head, intact. Deformed femoral heads are not measured as those are excluded from our study. 


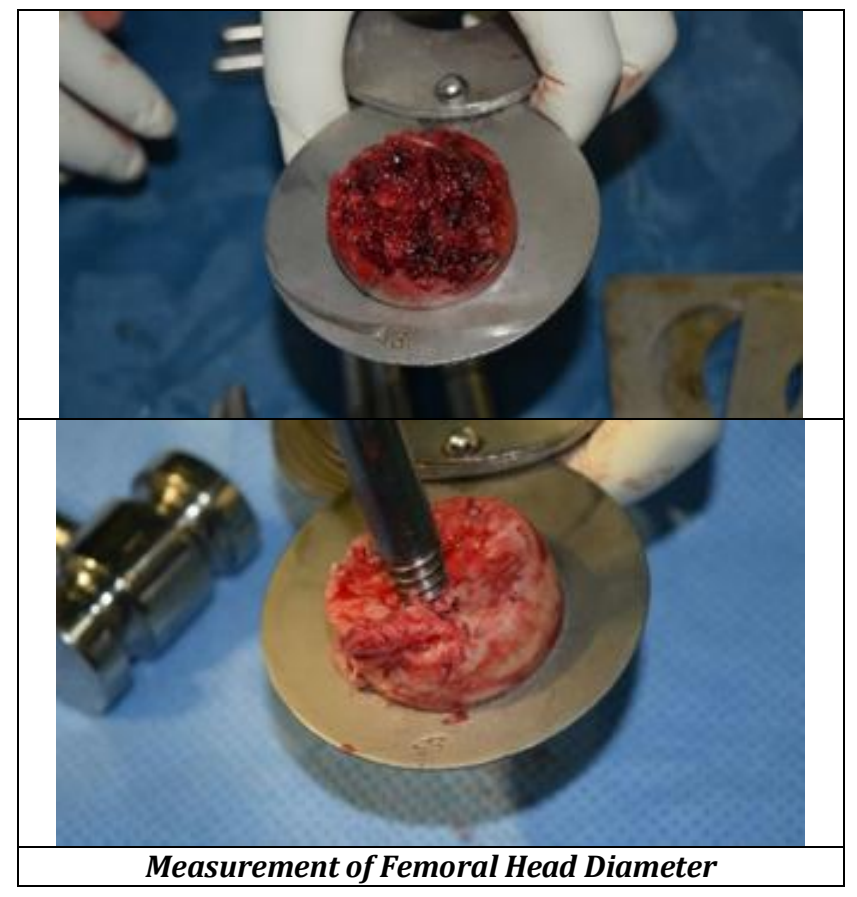

b) Distance between Tip of Greater Trochanter and Surface of Femoral Head: It was measure by Sliding calliper.(9) It was measured from tip of greater trochanter to a point along the axis of anteversion of femoral neck on the fovea of femoral head.

And after a simple calculation the distance from the centre of femoral head had achieved. In all the (fracture neck of femur) patients after anatomical reduction of head on neck was done and after proper holding it with k-wires or $3.2 \mathrm{~mm}$ or $2.5 \mathrm{~mm}$ cortical drill bits, the measurements were carried out.

Intraoperative distance of center of femoral head to tip of GT=tip of GT to a point at the fovea - (minus) radius of femoral head ( $1 / 2$ of diameter)

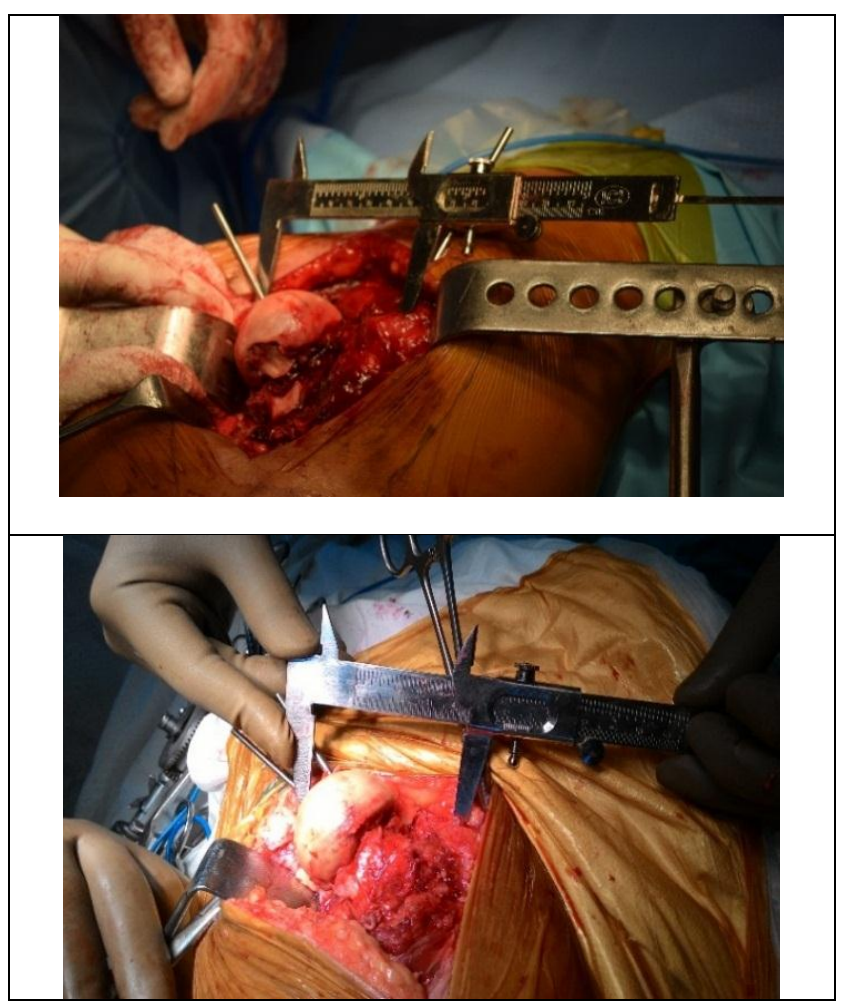

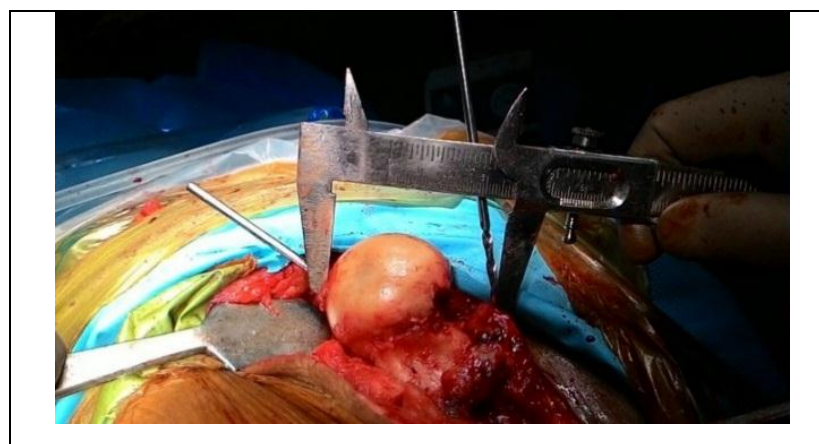

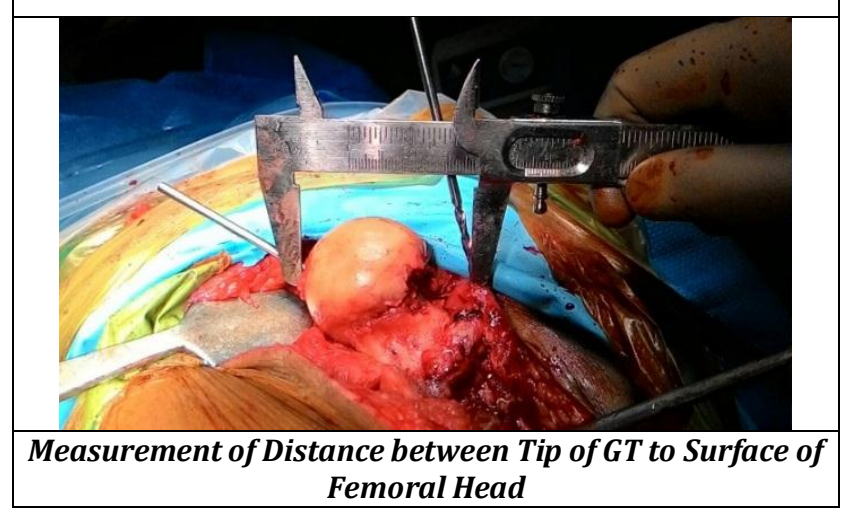

After taking all the measurements the hemiarthroplasty operation completed by standard technique with fixed bipolar, modular bipolar, Austin-Moore prosthesis with cemented or uncemented.

\section{A. Post-Operative Measurement}

In post-operative replaced or hemi replaced hip in x-ray of pelvis showing both hip AP in $15^{\circ}$ internal rotation(10) in true size film, measurement of distance between tip of GT to centre of femoral head of the prosthesis and in opposite normal side tip of GT to centre of femoral head was done.

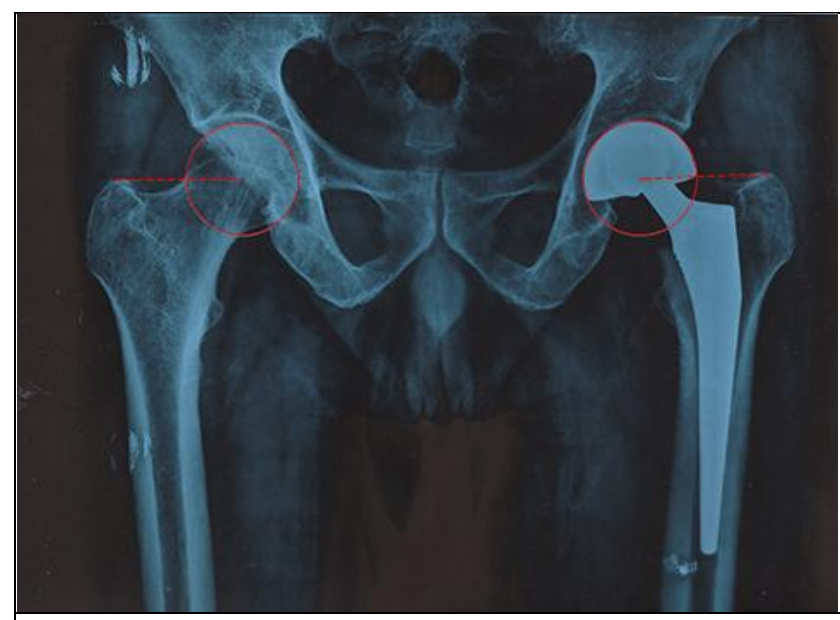

Postoperative True Size X Ray of Both Sides of Hip to Measure 1) Center of Prosthetic Femoral Head and Tip of GT 2) Center of Femoral Head of Normal Side and Tip of GT

\section{B. Outcomes}

The outcomes of the study measured under the following headings-

- Head of femur diameter.

- Distance of tip of GT to surface of femoral head (in the line of femoral anteversion the greatest distance was measured). 
- Distance of tip of GT to centre of head of femoral prosthesis in $\mathrm{X}$-ray.

- Distance of tip of GT to centre of head of femoral head of normal side in $x$-ray.

- Limb length discrepancy will be measured by measuring the distance between anterior superior iliac spine and tip of the medial malleolus and compared with the opposite side in identical position.

\section{Statistical Methods}

The statistical software SPSS version 20 has been used for the analysis. Categorical variables have been expressed as Number of patients and percentage of patients. Continuous variables have been expressed as Mean and Standard Deviation and Comparison between the groups was done by using paired $t$ test and independent $t$ test. $p$ value is less than 0.05 was considered as statistically significant.

\section{RESULTS}

\begin{tabular}{|c|c|}
\hline Variable & No (\%) \\
\hline Gender & \\
\hline Male & $22(41.5)$ \\
\hline Female & $31(58.5)$ \\
\hline Age (in years) & $73.5 \pm 8.3$ \\
\hline Approach & \\
\hline MHDG & $25(47.2)$ \\
\hline Post & $28(52.8)$ \\
\hline $\begin{array}{l}\text { Intra Operative Femoral head diameter } \\
\text { in } \mathrm{mm}\end{array}$ & $44.96 \pm 2.08$ \\
\hline $\begin{array}{l}\text { Intra Operative Tip of GT to femoral head } \\
\text { surface in mm }\end{array}$ & $76.70 \pm 2.33$ \\
\hline $\begin{array}{l}\text { Intra Operative Tip of GT to centre of femoral } \\
\text { head in } \mathrm{mm}\end{array}$ & $54.22 \pm 2.10$ \\
\hline $\begin{array}{l}\text { Post operative Tip of Gt to centre of head of } \\
\text { femoral prosthesis }\end{array}$ & $54.62 \pm 2.33$ \\
\hline $\begin{array}{l}\text { Post operative Tip of GT to centre of opposite } \\
\text { femoral head }\end{array}$ & $54.45 \pm 2.15$ \\
\hline LL of same side in $\mathrm{cm}$ & $87.38 \pm 3.69$ \\
\hline LL of opposite side in $\mathrm{cm}$ & $87.38 \pm 3.69$ \\
\hline $\begin{array}{c}\text { Gap between Post operative Tip of Gt to centre } \\
\text { of head of femoral prosthesis and Post } \\
\text { operative Tip of GT to centre of opposite } \\
\text { femoral head in mm }\end{array}$ & $-0.17 \pm 1.85$ \\
\hline $\begin{array}{c}\text { Gap between Post Operative and Intra } \\
\text { Operative Tip of GT to centre of femoral head in } \\
\mathrm{mm}\end{array}$ & $0.41 \pm 1.60$ \\
\hline
\end{tabular}

Comparison of different values was done between

1. Post-operative Tip of GT to center of head of femoral prosthesis AND intra-operative Tip of GT to center of femoral head.

2. Post-operative Tip of GT to center of opposite femoral head AND Intraoperative tip of GT to center of femoral head in MM.

3. Post- operative Tip of GT to center of opposite femoral head AND post operative tip of GT to center of Head of femoral prosthesis.

\begin{tabular}{|c|c|c|c|}
\hline 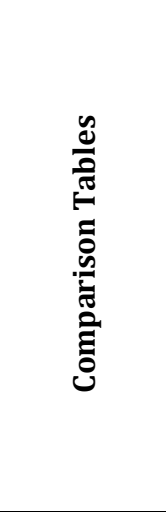 & $\begin{array}{l}\text { Post operative } \\
\text { Tip of Gt to } \\
\text { centre of head } \\
\text { of femoral } \\
\text { prosthesis - } \\
\text { Intra Operative } \\
\text { Tip of GT to } \\
\text { centre of } \\
\text { femoral head in } \\
\text { mm } \\
\text { (Compared by } \\
\text { paired-t test) }\end{array}$ & $\begin{array}{c}\text { Post operative } \\
\text { Tip of GT to } \\
\text { centre of } \\
\text { opposite } \\
\text { femoral head - } \\
\text { Intra } \\
\text { Operative Tip } \\
\text { of GT to centre } \\
\text { of femoral } \\
\text { head in mm } \\
\text { (Compared by } \\
\text { paired-t test) }\end{array}$ & \begin{tabular}{|c|} 
Post operative \\
Tip of GT to \\
centre of \\
opposite femoral \\
head - Post \\
operative \\
Tip of Gt to \\
centre of head of \\
femoral \\
prosthesis in \\
mm \\
(Compared by \\
Independent - $t$ \\
test) \\
\end{tabular} \\
\hline P value & & 0.007 & 0.483 \\
\hline nificance & ficant & Not Significant & Not Significant \\
\hline \multicolumn{4}{|c|}{ ble 2. Comparison between Different p-Values } \\
\hline
\end{tabular}

\section{DISCUSSION}

Displaced fractures of the femoral neck necessitate replacement surgery by using suitable prosthesis for early mobilization of the patients. But which prosthesis design is most suitable, especially in our eastern part of India is not known or studied. In our study we tried to measure that how much normal anatomy of the hip can be produced by total or hemi hip replacement. But during the specified study period we did not get any patient for total hip replacement, so all our patients were fracture neck of femur patients, who were treated by hemiarthroplasty. Here we didn't use any specific prosthesis design, but mostly we used fixed bipolar femoral prosthesis and modular bipolar femoral prosthesis, and only in one patient we used Austin Moore prosthesis. Here instead of conventional offset measurement we measure the distance between the two fixed bony points (Tip of GT and centre of femoral head) and compared the measurement of intra operative clinical value and post operative radiological value in true size x-rays and also with the opposite hip joint.

In our study we tried to find that is there any superiority of any specific prosthesis design over others in restoration normal anatomy and thus normal hip bio mechanics.

All the measurements were taken with consent of the patients who were treated for fracture neck of femur in Ramakrishna Mission Seva Pratishthan, Kolkata. There was no extra cost burden to the patient nor was any post operative morbidity occurred during the measurements.

53 patients were included in the study all belonging to more than 60 years of age regardless of their cast, religion, or geographic variation. There was no drop out from the study.

In our study we have found that minimum diameter of femoral head to be $41 \mathrm{~mm}$ and maximum $49 \mathrm{~mm} \mathrm{(44.96 \pm}$ $2.08 \mathrm{~mm}$ ). In the case of per operative distance between tip of greater trochanter and surface of the femoral head was obtained within a range of 72-82 mm (mean \pm SD: $76.70 \pm$ $2.33 \mathrm{~mm}$ ) and thus intra operative tip of greater trochanter to centre of rotation of femoral head distance, minimum was $48.5 \mathrm{~mm}$ while maximum was $58 \mathrm{~mm}$ (Mean \pm S.D.: $54.22 \pm$ $2.10 \mathrm{~mm}$ ). Post operative tip of greater trochanter to centre of head of femoral prosthesis distance in skiagram, minimum was $50 \mathrm{~mm}$ while maximum was $60 \mathrm{~mm}$ (Mean \pm S.D.: $54.22 \pm$ $2.63 \mathrm{~mm}$ ). Post operative tip of greater trochanter to centre of femur in opposite side in skiagram, minimum was $49 \mathrm{~mm}$ while maximum was $59 \mathrm{~mm}$ (Mean \pm S.D.: $54.45 \pm 2.15 \mathrm{~mm}$ ). 
In our study we found that, the difference between the values of post operative tip of GT to centre of head of femoral prosthesis in x-rays and intra operative tip of GT to centre of femoral head measured clinically, was as minimum as (-) 3 $\mathrm{mm}$ and as maximum as $2.5 \mathrm{~mm}$ (Mean \pm S.D.: $0.41 \pm 1.60$ ). Which shows the p-value of 0.061 , which means there is no significant difference between intra and post operative measurements. Which means that we reproduced the normal anatomy after the replacement significantly.

The difference between the values of post operative tip of GT to centre of head of femoral prosthesis and post operative tip of GT to centre of femoral head in opposite side showed as minimum as $(-) 4 \mathrm{~mm}$ and as maximum as $4 \mathrm{~mm}$ (Mean \pm S.D.: $0.17 \pm 1.85 \mathrm{~mm}$ ). Which had the $\mathrm{p}$-value of 0.069 , means there is no significant difference between the replaced hip with the opposite one. Which indicate that we had measured the anatomy successfully.

The difference between the values of postoperative tip of GT to centre of femoral head in opposite side with postoperative tip of GT to centre of head of femoral prosthesis showed that $\mathrm{p}$-value is 0.483 . Which means that there is no significant difference between these two measurements, done in true sized postoperative x-ray. That indicates we successfully recreate the anatomy of the hip as compared with opposite side.

In our study we found that there is no significant difference in the parameters with age, sex, side, approach (Posterior Approach with modified Hardinge Approach), cementation (uncemented with cemented).

Contrary to our belief we found that there is so significant difference in results with different prosthesis design. Here we used modular bipolar prosthesis (MBP) in 20 patients and fixed bipolar prosthesis (FBP) in 32 patients. The difference between Postoperative Tip of Gt to centre of head of femoral prosthesis and Postoperative Tip of GT to centre of opposite femoral head in FBP is mean \pm SD: $-0.24 \pm 2.02$. And the difference between Postoperative Tip of Gt to centre of head of femoral prosthesis and Postoperative Tip of GT to centre of opposite femoral head in MBP is mean \pm SD: $-0.5 \pm 1.57$ with p-value of 0.628. Gap between Postoperative GT to centre of prosthesis head and Intra Operative Tip of GT to centre of femoral head in mm showed in FBP mean \pm SD: $0.42 \pm 1.65$ and in MBP Mean \pm S.D.: $0.38 \pm 1.54$ with $\mathrm{p}$-value of 0.838 . Thus, it is found that there is no superiority -of MBP over FBP. It may be due to the fact that though we used MBP, in almost every patient we used the zero (" 0 ") neck size. Or it may be due to small study population and may shows different result with larger no of population.

When we compared our study with other studies by different authors following results we have found and have tabulated in a comparative manner:

From the above comparison we found that the head diameter of Siwach and Dahiya were close to the head diameter we measured. And the limb length discrepancy found by Eggil et al in preoperative and postoperative had a significant difference with our study. This is because they took limb length in fraction of $\mathrm{cm}$ but we took it to the nearest $\mathrm{cm}$.

Our study didn't show any superiority of one prosthesis design over another in cases of recreating the normal anatomy of hip joint with femoral prosthesis.

\section{Recommendations}

A. Larger sample size with multi-centric study will produce more accurate comparison.

B. Team work with radiologists and anthropometrics measurements in cadaver will enrich our knowledge of proximal femoral anatomy.

C. Development of some device by bio-medical engineers can simplify these measurements per-operatively.

D. Data of the same prosthesis designs (FBP or MBP) of different maker will enrich our study.

E. Variations with height, weight, race and co-relation with functional outcome can make our study more informative.

\section{Review of Literature}

1. The tip of the greater trochanter a reliable reference for the rotation centre of the femoral head.(11) One study analysed standard anterior-posterior pelvic radiographs of 225 patients with osteoarthritis of the hip who were about to undergo total hip arthroplasty. The distance between the tip of the greater trochanter and the rotation centre of the femoral head was measured for the affected hip.

It was found that, the average location of the tip of greater trochanter is $3.4 \mathrm{~mm}$ proximal to the centre of the femoral head, with a range from $20 \mathrm{~mm}$ proximal to $10 \mathrm{~mm}$ distal to the femoral head centre. This study was done in by Kanthan Theivendran et al, From New Cross Hospital, Wolverhampton.

2. One study showed the importance of preoperative templating in THR. They analysed the value and accuracy of preoperative planning for total hip replacement (THR) (12). They digitised electronically and compared the handsketched preoperative plans with the pre- and postoperative radiographs of 100 consecutive primary THRs.

The correct type of prosthesis was planned in 98\%; the agreement between planned and actually used components was $92 \%$ on the femoral side and $90 \%$ on the acetabular side. The mean $( \pm \mathrm{SD}$ ) absolute difference between the planned and actual position of the centre of rotation of the hip was $2.5 \pm 1.1 \mathrm{~mm}$ vertically and $4.4 \pm$ $2.1 \mathrm{~mm}$ horizontally. On average, the inclination of the acetabular component differed by $7 \pm 2^{\circ}$ and anteversion by $9 \pm 3^{\circ}$ from the

Preoperative plans. The mean postoperative leg-length difference was $0.3 \pm 0.1 \mathrm{~cm}$ clinically and $0.2 \pm 0.1 \mathrm{~cm}$ radiologically. More than $80 \%$ of intraoperative difficulties were anticipated. Preoperative planning is of significant value for the successful performance of THR. This study was done by, Eggli et al, From the University of Bern.

3. One study showed detailing of proximal femoral anatomy in total hip arthroplasty (THA).(13) They performed tri-planar computed tomography analysis of the proximal femoral anatomy in a series of 150 patients ( $n=150)$ to accurately delineate this relationship. The mean location for the centre of the Femoral Head (FH) was $8.64 \mathrm{~mm}$ (95\% confidence interval, 9.44-7.83) distal to the tip of the GT. The centre of the FH was found to be distal to the tip of the GT in $90.6 \%$ of cases. Hence they suggest caution in using the tip of the GT as a reference 
point during total hip arthroplasty as it could be associated with an inadvertent intraoperative leg lengthening. This study was done by Adeel Rasool Memon et al, from Cork University Hospital.

4. For per operative restoration of limb-length and femoral offset, there was a study done in France. They made a mechanical measurement device (length and offset optimization device [LOOD]) fixed to the pelvis can optimize lower-limb length and offset control during THA performed on a posterolateral approach.

Two prospective THA series were compared: 32 using the LOOD and 26 without. Patients with more than $5 \mathrm{~mm}$ preoperative limb-length discrepancy were excluded. The intraoperative target was to restore individual anatomy. Radiographic analysis was based

On pre- and postoperative AP pelvic weight-bearing views in upright posture, feet aligned, with comparison to per operative LOOD data.

Mean deviation from target length (i.e., pre- to postoperative length differential) was $2.31 \mathrm{~mm}$ (range, $0.04-10.6 \mathrm{~mm}$ ) in patients operated on using the LOOD versus $6.96 \mathrm{~mm}(0.01-178 \mathrm{~mm})$ without LOOD $(\mathrm{P}=$ $0.0013)$. Mean deviation from target offset was 3.96 (0.45-13.50) $\mathrm{mm}$ with LOOD versus 10.16 (0.93-28.81) without $(\mathrm{P}=0.0199)$. There was no significant difference between operative and radiographic measurements of length deviation using LOOD $(\mathrm{P}=0.4)$; those for offset, however, differed significantly ( $\mathrm{P}=0.02)$. This study was done by, O. Barbier et al, Military Teaching Hospital, 69, avenue de Paris, 94160 Paris.(14)

5. A study was conducted by Siwach and Dahiya from Dept of Orthopaedics, Postgraduate Institute of Medical Sciences, Rohtak, India on 150 adult cadaveric femoral head (Age approximately between 20 year to 80 years). The study was on the anthropometric measurement of proximal femur geometry and its clinical significance. 75 pairs of cadaveric femur were studied morphologically and radio logically using standard technique to obtain the following measurements. (i) femoral head offset mean $43.95 \mathrm{~mm}$ with SD 3.06, (ii) femoral head diameter mean $43.53 \mathrm{~mm}$ with SD 3.40, (ii) femoral neck diameter, (iii) canal width at and $20 \mathrm{~mm}$ above and below LT (iv) endosteal and extra cortical width at the isthmus (v) femoral neck anteversion, neck shaft angle. After calculating the mean value and standard deviations, the measurements were used to determine femoral head volume, and the cross sectional area of femoral neck. Also, the percentage of neck occupied by various implants was observed. These values were compared with the results reported in literature published in Hong Kong, China. Caucasian and western data was found to be quite different. The impact of these finding on future implant design were discussed.

\section{CONCLUSION}

Our study results showed that there is no significant difference in various prosthesis designs for replacement of femoral component. All the prosthesis designs, such as FBP or MBP are similarly effective for recreating the hip anatomy; which means that all prosthesis designs from different makers are suitable for Indian population. There is no significant limb length discrepancy between the operated sides with opposite side. This data will help in the decision making about the amount of neck cutting during total or hemi-replacement of hip. In our study, we didn't address the different makers, nor did we use prosthesis with same design or of same makers. As our study period was only one year and sample size was only 53 , there is a need for larger studies with larger sample size including patients with racial, geographical variations and correlations with height and weight.

\section{REFERENCES}

[1] Standring S. Gray's Anatomy: the anatomical basis of clinical practice. $40^{\text {th }}$ edn. Churchill Livingstone Elsevier 2008;81:1379-80.

[2] Bowman KF Jr, Fox J, Sekiya JK. A clinically relevant review of hip biomechanics. Arthroscopy 2010;26(8):1118-29.

[3] Loughead JM, Chesney D, Holland JP, et al. Comparison of offset in Birmingham hip resurfacing and hybrid total hip arthroplasty. J Bone \& Joint Surgery $\mathrm{Br}$ 2005;87(2):163-6.

[4] Kjellberg M, Englund E, Sayed-Noor AS. A new radiographic method of measuring femoral offset. The Sundsvall method. Hip Int 2009;19(4):377-81.

[5] Sarin VK, Pratt WR, Bradley GW. Accurate femur repositioning is critical during intraoperative total hip arthroplasty length and offset assessment. The Journal of Arthroplasty 2005;20(7):887-91.

[6] Antapur P, Prakash D. Proximal femoral geometry: a radiological assessment. J Arthroplasty 2006;21(6):897-8.

[7] Siwach RC, Dahiya S. Anthropometric study of proximal femur geometry and its clinical application. Ind J Orthopaedics 2003;37(4):247-51.

[8] Athapattu M, Saveh AH, Mahmud J, et al. Accuracy of measuring methods on the femoral head. Procedia Engineering 2013;68:83-7.

[9] Jeffery JA, Ong TJ. Femoral head measurement in hemiarthroplasty: assessment of interobserver error using 3 measuring systems. Injury 2000;31(3):135-8.

[10] Clohisy JC, Carlisle JC, Beaulé PE, et al. A systematic approach to the plain radiographic evaluation of the young adult hip. J Bone Joint Surg Am 2008;90 Suppl 4:47-66.

[11] Theivendran $\mathrm{K}$, Hart WJ. Is the tip of the greater trochanter a reliable reference for the rotation centre of the femoral head in total hip arthroplasty? Acta Orthop Belg 2009;75(4):472-6.

[12] Eggli S, Pisan M, M"uller ME. The value of preoperative planning for total hip arthroplasty. J Bone Joint Surg $\mathrm{Br}$ 1998;80(3):382-90.

[13] Memon AR, Butler J, Guerin S, et al. Proximal femoral anatomy in total hip arthroplasty. A tri-planar computed tomographic assessment. Acta Orthop Belg 2011;77(4):488-93.

[14] Barbier O, Ollat D, Versier G. Interest of an intraoperative limb-length and offset measurement device in total hip arthroplasty. Orthop Traumatol Surg Res 2012;98(4):398-404. 